\title{
Maximizing the Power Transfer for a Mixed Inductive and Capacitive Wireless Power Transfer System
}

\author{
Ben Minnaert and Nobby Stevens \\ KU Leuven, DRAMCO, Department of Electrical Engineering (ESAT) \\ Ghent Technology Campus, 9000 Ghent, Belgium \\ ben.minnaert@kuleuven.be; nobby.stevens@kuleuven.be
}

\begin{abstract}
Nowadays, near-field wireless power transfer is realized by inductive or capacitive coupling. Power transmission is accomplished by a time-varying magnetic or electric field as medium, respectively. Recently, mixed or hybrid wireless power transfer is being developed as a possible mean to increase the power density of the system by utilizing both the magnetic and the electric near-field. The fundamental basics of mixed coupling are well understood. However, the implications of the mixed coupling theory on wireless power transfer applications is not rigorously described. Moreover, no general description is available that allows for a detailed comparison between current hybrid systems, especially for a series topology of inductive and capacitive coupling. In this work, we analytically solve a general mixed wireless power transfer configuration with series topology. We determine the optimal load to maximize the amount of power transfer and calculate the maximum achievable output power. The analytical derivation is validated by numerical simulation in SPICE. Our solution allows for a better fundamental understanding of the mixed wireless link and can serve as a reference point to evaluate the performance of mixed systems with regard to power transfer.
\end{abstract}

Index Terms - capacitive power transmission, electromagnetic coupling, hybrid coupling, inductive power transmission, mixed coupling, mutual coupling, wireless power transmission.

\section{INTRODUCTION}

Near-field wireless power transfer (WPT) across an air gap can be realized by coupled resonators. Two different principles exist:

- Inductive wireless power transfer (IPT) uses coupled inductors to transfer energy from a transmitter coil to a receiver coil. Power transfer is accomplishes by a timevarying magnetic field as medium [1].

- Capacitive wireless power transfer (CPT) applies coupled capacitances for the power transfer from a transmitter plate to a receiver plate. The medium that realizes the power transfer is a time-varying electric field. CPT can be seen as the dual technique of IPT [2].

The IPT technology can be considered as relatively mature. Therefore, a large range of IPT applications have already entered the market [1]. Most known are portable electronics and household devices (e.g., electric toothbrushes, smartphones, smart watches and wearables). In the higher power range, IPT is applied to industrial automation (e.g., automated guided vehicles and robots) and electric vehicles.

The CPT technology is lagging behind compared to IPT. Research and development efforts for CPT are increasing, in particular for electric vehicles applications [3]. As far as we

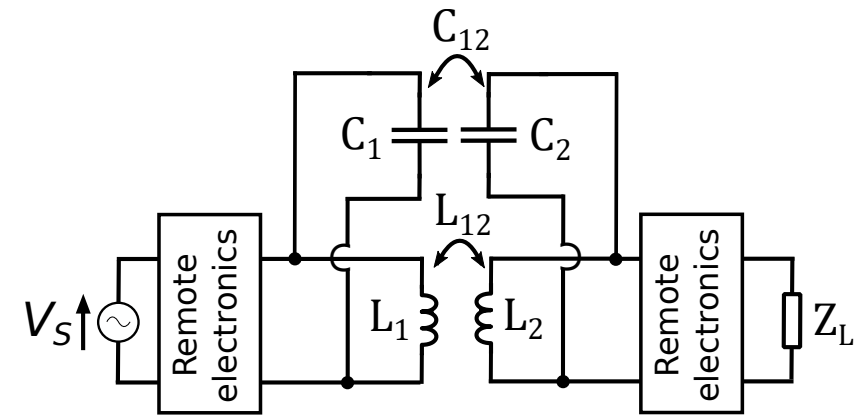

Fig. 1. Schematic circuit overview of a general mixed WPT system with IPT and CPT in parallel topology.

know, no commercial CPT applications are available on the market today.

Recently, mixed (also called hybrid) coupling is being considered by the research community as a possible mean to realize electromagnetic wireless power transfer [4]-[9]. Mixed coupling uses as well the magnetic as the electric near-field to transfer power. Both coupled inductors and capacitors are applied in transmitter and receiver. Consider for example Fig. 1. Power is wirelessly transferred from the voltage source $V_{S}$ to the load $Z_{L}$ by the coupled inductors $L_{1}$ and $L_{2}$ with mutual inductance $L_{12}$, and by the coupled capacitances $C_{1}$ and $C_{2}$ with mutual capacitance $C_{12}$ [10]. Remote electronics (e.g., the compensation circuits, power converter and rectifier) are present in a practical WPT system to create resonance and improve the power transfer.

There are several arguments why a mixed or hybrid system could for certain applications be preferable to a single IPT or CPT system:

- It is possible to increase the amount of power transfer of an IPT or CPT system by enlarging the coil or capacitor plate area, respectively. However, in practical applications, this option is often not feasible due to size restrictions (e.g., smart watch, small sensor networks,... ). Applying as well the magnetic as the electric field as medium for WPT allows for a larger amount of power transfer within the same space, or for a smaller transmitter and receiver area for the same amount of power transfer [4].

- In order to create a resonant circuit, different topologies are possible for an IPT system, depending on the ap- 


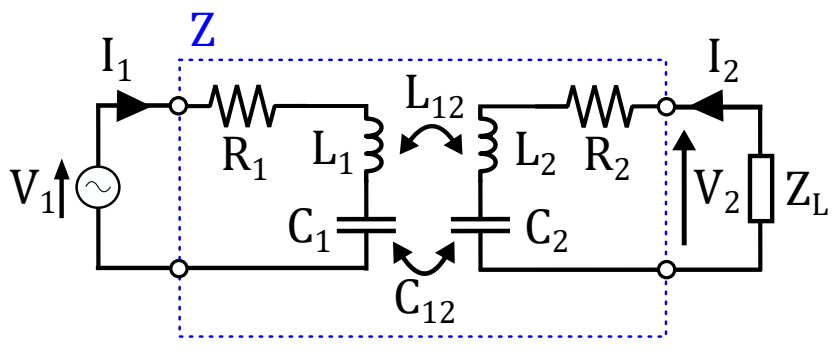

Fig. 2. Equivalent network of a mixed WPT setup, where IPT and CPT are arranged in series. The wireless link can be considered as a two-port network, characterized by its impedance matrix $\mathbf{Z}$.

plication. However, all those topologies use at least one external capacitor. Analogous, any practical CPT system uses at least one external inductor to realize the resonance for WPT. Since any near-field WPT system applies at transmitter and receiver side at least both inductors and capacitances, it can be meaningful to apply mixed coupling to utilize the potential of both inductive and capacitive components [5], [6]. As a result, an increased power density for the entire WPT system can be achieved, fully using the circuit components and both magnetic and electric fields.

- Applying the different properties of magnetic and electric coupling, a mixed WPT system could allow for a coupling-independent regime at a fixed operating frequency [7]. In this way, the WPT system could become more robust against lateral misalignment and varying vertical displacements [8].

The fundamental basics of mixed coupling are well understood [10]. However, the implications of the mixed coupling theory on WPT applications is not rigorously described. Moreover, no general WPT description is available that allows for a detailed comparison between current hybrid systems. The scientific literature [4], [9]-[11] describes mixed WPT systems where IPT and CPT are arranged in a parallel topology as in Fig. 1. However, the experimental set-ups currently built in research labs do not apply the parallel topology, but a series topology as shown in Fig. 2. For example, Lu et al. [5] built a mixed $3.0 \mathrm{~kW}$ WPT system for the charging of electric vehicles. They apply a series topology of IPT and CPT to obtain a system efficiency of $94.5 \%$ at an operating frequency of $1 \mathrm{MHz}$. The same group also developed a $100 \mathrm{~W}$ prototype with a novel coupler structure [6] that allows for mixed WPT at $73.6 \%$. Their coupler structure consists of a metallic grid and can be seen as an IPT and CPT coupling in series. Also Zhang et al. [7] applied the series topology for a distanceinsensitive mixed WPT setup operating at efficiencies of $75 \%$ and higher.

The aforementioned examples study in detail their specific set-up, but these analysis are not applicable to a general mixed set-up. In this work, we rigorously solve a general mixed WPT configuration where the magnetic and electric couplings are arranged in series. Our solution allows for a better fundamental understanding of the mixed wireless link for WPT applications. More specifically, our contributions are the following:

- The optimal load for a mixed WPT system with series topology is determined.

- The maximum achievable power transfer is calculated to serve as a reference point for evaluating the performance of mixed WPT systems.

- The analytical derivation is validated by numerical simulation in SPICE.

\section{Methodology}

\section{A. Circuit analysis}

Consider a general mixed WPT system, given by Fig. 2 . Notice that the coupled inductors and coupled capacitors are arranged in series. The magnetic coupling factor $k_{m}$ and electric coupling factor $k_{e}$ are a measure for the strength of each coupling and are defined by [10]:

$$
\begin{aligned}
& k_{m}=\frac{L_{12}}{\sqrt{L_{1} L_{2}}} \\
& k_{e}=\frac{C_{12}}{\sqrt{C_{1} C_{2}}}
\end{aligned}
$$

In a practical WPT system, the polarity of the coupled coils will be arranged in such a way that the IPT will enhance to the CPT. An opposite polarity could cancel out or decrease the CPT contribution. The WPT system is supplied by a voltage source with as peak-value voltage phasor $V_{1}$ and operating angular frequency $\omega_{0}$. The resistive losses at transmitter and receiver side are represented by $R_{1}$ and $R_{2}$, respectively. The inductors and capacitors are chosen such that a resonant circuit is created:

$$
\omega_{0}=\frac{1}{\sqrt{L_{1} C_{1}}}=\frac{1}{\sqrt{L_{2} C_{2}}}
$$

We consider the wireless link as a two-port network, fully characterized by its impedance matrix $\mathbf{Z}$, given by:

$$
\mathbf{Z}=\left[\begin{array}{cc}
R_{1}+j \omega L_{1}+\frac{C_{2}}{j \omega \gamma_{c}} & j \omega L_{12}+\frac{C_{12}}{j \omega \gamma_{c}} \\
j \omega L_{12}+\frac{C_{12}}{j \omega \gamma_{c}} & R_{2}+j \omega L_{2}+\frac{C_{1}}{j \omega \gamma_{c}}
\end{array}\right]
$$

with

$$
\gamma_{c}=C_{1} C_{2}-C_{12}^{2}
$$

The current-voltage relation of the two-port network is given by:

$$
\left[\begin{array}{l}
V_{1} \\
V_{2}
\end{array}\right]=\mathbf{Z}\left[\begin{array}{l}
I_{1} \\
I_{2}
\end{array}\right]
$$

with $V_{i}$ and $I_{i}(i=1,2)$ the peak voltage and current phasors, respectively, as defined on Fig. 2. 


\section{B. Power maximization}

For the general mixed WPT circuit of Fig. 2, we determine analytically the optimal load that maximizes the power transfer. The power $P_{L}$ dissipated by the load $Z_{L}=R_{L}+j X_{L}$ is given by:

$$
P_{L}=\frac{\left|V_{2}\right|^{2}}{2 R_{L}}
$$

For convenience, we define the following parameters:

$$
\begin{aligned}
a_{1} & =\gamma_{c} L_{1} \omega^{2}-C_{2} \\
a_{12} & =\gamma_{c} L_{12} \omega^{2}-C_{12} \\
a_{2} & =\gamma_{c} L_{2} \omega^{2}-C_{1} \\
b & =\gamma_{c} R_{1} \omega
\end{aligned}
$$

In order to find the optimal load, we calculate the Thévenin equivalent as seen from the input port. The Thévenin voltage $V_{t h}$ is equal to the open circuit voltage at the output port of the two-port network. From (6), with $I_{2}=0$ and $V_{2}=V_{t h}$, we obtain:

$$
V_{t h}=\frac{a_{1}+j b}{a_{1}^{2}+b^{2}} a_{12} V_{1}
$$

The Thévenin impedance $Z_{t h}$ is equal to $Z_{t h}=V_{2} / I_{2}$ for the two-port network with $V_{1}=0$. From (6), we find:

$$
Z_{t h}=\frac{a_{12}^{2}}{a_{1}^{2}+b^{2}} R_{1}+R_{2}+j \frac{a_{1} d+a_{2} b}{\gamma_{c} \omega\left(a_{1}^{2}+b^{2}\right)}
$$

with

$$
\begin{aligned}
d=\left(L_{1} L_{2}-\right. & \left.L_{12}^{2}\right) \gamma_{c}^{2} \omega^{4} \\
& -\left(C_{1} L_{1}+C_{2} L_{2}-2 C_{12} L_{12}\right) \gamma_{c} \omega^{2}+\gamma_{c}
\end{aligned}
$$

The load $Z_{L}^{\prime}=R_{L}^{\prime}+j X_{L}^{\prime}$ that maximizes the power $P_{L}$ is the complex conjugate of $Z_{t h}$, due to the maximum power transfer theorem [12]. We obtain:

$$
\begin{gathered}
R_{L}^{\prime}=\frac{a_{12}^{2}}{a_{1}^{2}+b^{2}} R_{1}+R_{2} \\
X_{L}^{\prime}=-\frac{a_{1} d+a_{2} b^{2}}{\gamma_{c} \omega\left(a_{1}^{2}+b^{2}\right)}
\end{gathered}
$$

Notice that a non-zero reactance is necessary to maximize the power transfer to the load. The reactance $X_{L}^{\prime}$ compensates the complex part of the two-port network input impedance. Applying the load $Z_{L}^{\prime}$ results in a purely resistive input impedance at the input port.

A positive reactance $X_{L}^{\prime}$ corresponds to an inductor $L_{L}^{\prime}$, whereas a negative reactance $X_{L}^{\prime}$ corresponds with a capacitor $C_{L}^{\prime}$, with values:

$$
\begin{gathered}
L_{L}^{\prime}=\frac{X_{L}^{\prime}}{\omega_{0}} \\
C_{L}^{\prime}=-\frac{1}{\omega_{0} X_{L}^{\prime}}
\end{gathered}
$$

The maximum output power $P_{L, \max }$ that a general mixed WPT system in series topology can achieve, can now be easily
TABLE I

SiMULATION VALUES.

\begin{tabular}{|c|c|}
\hline Quantity & Value \\
\hline$f$ & $100 \mathrm{kHz}$ \\
\hline$V_{1}$ & $1.00 \mathrm{~V}$ \\
\hline$R_{1}$ & $1.00 \Omega$ \\
\hline$R_{2}$ & $1.00 \Omega$ \\
\hline$L_{1}$ & $100 \mu \mathrm{H}$ \\
\hline$L_{2}$ & $100 \mu \mathrm{H}$ \\
\hline$L_{12}$ & $20.0 \mu \mathrm{H}$ \\
\hline$C_{1}$ & $25.3 \mathrm{nF}$ \\
\hline$C_{2}$ & $25.3 \mathrm{nF}$ \\
\hline$C_{12}$ & $2.53 \mathrm{nF}$ \\
\hline$k_{m}$ & $20.0 \%$ \\
\hline$k_{e}$ & $10.0 \%$ \\
\hline
\end{tabular}

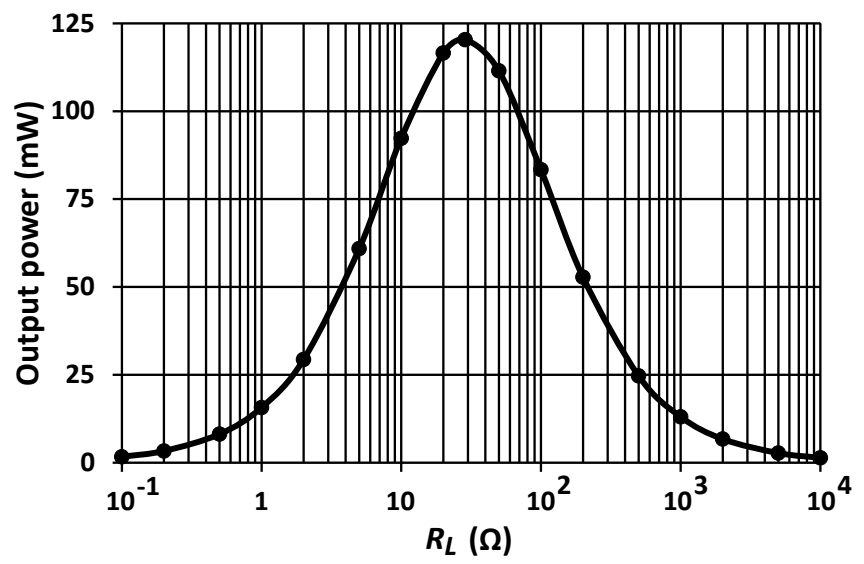

Fig. 3. The simulated output power for varying resistive load $R_{L}$. The load reactance remains fixed at $C_{L}^{\prime}=94.2 \mathrm{nF}$.

calculated. With the optimal load $Z_{L}^{\prime}$ applied to the two-port network, from (7) and the Thévenin circuit, we obtain:

$$
P_{L, \max }=\frac{a_{12}^{2}\left|V_{1}\right|^{2}}{8 R_{1} a_{12}^{2}+8 R_{2}\left(a_{1}^{2}+b^{2}\right)}
$$

This expression can serve as a reference point to evaluate the performance of specific mixed WPT systems with regard to power transfer and allows comparison between different systems.

\section{NUMERICAL VALIDATION}

We validate the analytical calculation by circuit simulation in SPICE. We consider the circuit of Fig. 2. We assume the numerical values given in Table I.

Calculation from (15) and (16) results in an optimal load $Z_{L}^{\prime}=R_{L}^{\prime}+j X_{L}^{\prime}$ for maximizing the power transfer of the system. We find $R_{L}^{\prime}=28.7 \Omega$ and $X_{L}^{\prime}=-16.9 \Omega$. The negative complex part of the load corresponds with a capacitor $C_{L}^{\prime}$ equal to $94.2 \mathrm{nF}$. By applying this load to the mixed network, the maximum output power $P_{L, \max }$ is achieved. From (19), we compute $P_{L, \max }=121 \mathrm{~mW}$.

First, we simulate the circuit in SPICE for varying load resistance. Fig 3 shows the output power dissipated by the 


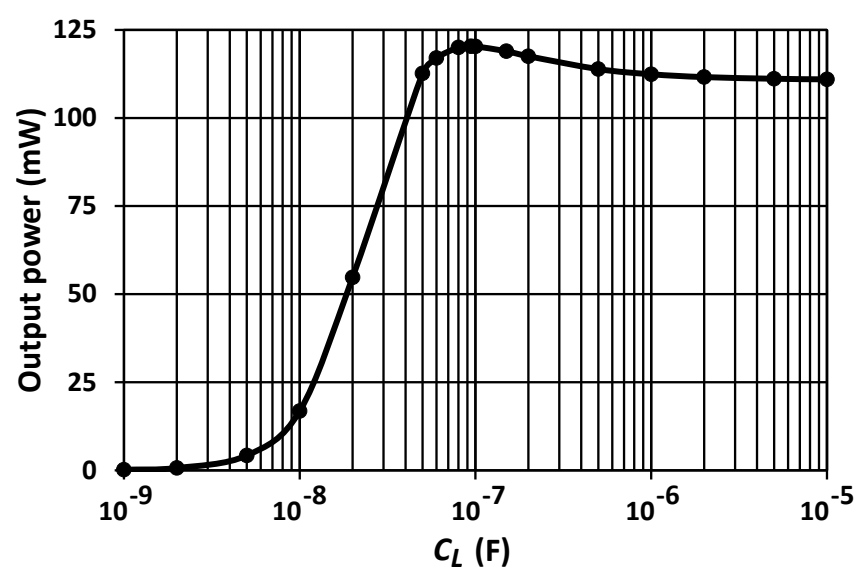

Fig. 4. The simulated output power for varying reactive load, i.e. varying capacitor $C_{L}$. The load resistance remains fixed at $R_{L}^{\prime}=28.7 \Omega$.

load. The load reactance is kept fixed at $C_{L}^{\prime}=94.2 \mathrm{nF}$. We observe that the maximum output power is achieved at the analytically calculated optimal resistive load of $R_{L}^{\prime}=28.7 \Omega$, and corresponds with the calculated value of $121 \mathrm{~mW}$.

Secondly, we perform the simulation for varying load reactance. Now, we keep the resistive part of the load fixed at its optimal value of $R_{L}^{\prime}=28.7 \Omega$. Fig 4 shows the result. Again, a perfect correspondence between simulation and the analytical derivation can be found: a maximum power transfer occurs at a capacitance of $C_{L}^{\prime}=94.2 \mathrm{nF}$. Notice that for this example, the output power remains high for capacitances larger than $C_{L}^{\prime}$.

\section{CONCLUSION}

We determined the optimal load for a mixed WPT system with series topology. We found that a non-zero reactance is necessary to compensate for the complex input impedance of the system. The maximum achievable power transfer was calculated to serve as a reference point for evaluating the performance of mixed WPT systems. The analytical calculations were numerically validated by SPICE simulations. Our solution can be seen as a first step to a rigorous description of the mixed coupling theory for mixed WPT applications.

\section{REFERENCES}

[1] X. Lu, P. Wang, D. Niyato, D. I. Kim, and Z. Han, "Wireless charging technologies: Fundamentals, standards, and network applications," IEEE Communications Surveys \& Tutorials, vol. 18, no. 2, pp. 1413-1452, 2016.

[2] B. Minnaert and N. Stevens, "Conjugate image theory applied on capacitive wireless power transfer," Energies, vol. 10, no. 1, p. 46, 2017.

[3] F. Lu, H. Zhang, and C. Mi, "A review on the recent development of capacitive wireless power transfer technology," Energies, vol. 10, no. 11, p. $1752,2017$.

[4] X. Chen, S. Yu, and X. Yang, "Hybrid wireless power transfer," IECON 2017 - 43rd Annual Conference of the IEEE Industrial Electronics Society, pp. 5348-5352, 2017.

[5] F. Lu, H. Zhang, H. Hofmann, and C. C. Mi, "An inductive and capacitive combined wireless power transfer system with LC-compensated topology," IEEE Transactions on Power Electronics, vol. 31, no. 12, pp. 8471-8482, 2016.

[6] F. Lu, H. Zhang, H. Hofmann, and C. C. Mi, "An inductive and capacitive integrated coupler and its LCL compensation circuit design for wireless power transfer," IEEE Transactions on Industry Applications, vol. 53, no. 5, pp. 4903-4913, 2017.

[7] X. Y. Zhang, C.-D. Xue, and J.-K. Lin, "Distance-insensitive wireless power transfer using mixed electric and magnetic coupling for frequency splitting suppression," IEEE Transactions on Microwave Theory and Techniques, vol. 65, no. 11, pp. 4307-4316, 2017.

[8] D. Vincent, P. H. Sang, and S. S. Williamson, "Feasibility study of hybrid inductive and capacitive wireless power transfer for future transportation," in Transportation Electrification Conference and Expo (ITEC), 2017 IEEE. IEEE, 2017, pp. 229-233.

[9] B. Minnaert and N. Stevens, "Conjugate image theory for non-symmetric inductive, capacitive and mixed coupling," in Wireless Power Transfer Conference (WPTC), 2017 IEEE. IEEE, 2017, pp. 1-4.

[10] J.-S. G. Hong and M. J. Lancaster, Microstrip filters for RF/microwave applications. John Wiley \& Sons, 2004, vol. 167.

[11] H. Hirayama, T. Amano, N. Kikuma, and K. Sakakibara, "Undesired emission and biological effect of open-end and short-end antennas for coupled-resonant wireless power transfer," in Electromagnetic Compatibility (APEMC), 2013 Asia-Pacific Symposium on. IEEE, 2013, pp. $1-4$.

[12] A. Bhaya, E. A. Herrera, and O. Diene, "Revisiting the maximum power transfer for linear n-ports with uncoupled loads and applications to power systems," International Journal of Circuit Theory and Applications, vol. 44, no. 9, pp. 1631-1656, 2016. 